\title{
Drawing Shortest Paths in Geodetic Graphs
}

\author{
Sabine Cornelsen ${ }^{1[0000-0002-1688-394 X]}$, \\ Maximilian Pfister 2[0000-0002-7203-0669], Henry Förster 2[0000-0002-1441-4189], \\ Martin Gronemann ${ }^{3}[0000-0003-2565-090 X]$, \\ Michael Hoffmann ${ }^{4}[0000-0001-5307-7106]$, \\ Stephen Kobourov ${ }^{5}[0000-0002-0477-2724]$, and \\ Thomas Schneck ${ }^{2}[0000-0003-4061-8844]$ \\ 1 University of Konstanz, Germany sabine.cornelsen@uni-konstanz.de \\ 2 University of Tübingen, Germany \\ \{pfister,foersth, schneck\}@informatik.uni-tuebingen.de \\ 3 University of Osnabrück, Germany \\ martin.gronemann@uni-osnabrueck.de \\ 4 Department of Computer Science, ETH Zürich, Switzerland \\ hoffmann@inf .ethz.ch \\ 5 Department of Computer Science, University of Arizona, USA \\ kobourov@cs.arizona.edu
}

\begin{abstract}
Motivated by the fact that in a space where shortest paths are unique, no two shortest paths meet twice, we study a question posed by Greg Bodwin: Given a geodetic graph $G$, i.e., an unweighted graph in which the shortest path between any pair of vertices is unique, is there a philogeodetic drawing of $G$, i.e., a drawing of $G$ in which the curves of any two shortest paths meet at most once? We answer this question in the negative by showing the existence of geodetic graphs that require some pair of shortest paths to cross at least four times. The bound on the number of crossings is tight for the class of graphs we construct. Furthermore, we exhibit geodetic graphs of diameter two that do not admit a philogeodetic drawing.
\end{abstract}

Keywords: Edge crossings · Unique Shortest Paths · Geodetic graphs.

\section{Introduction}

Greg Bodwin [1] examined the structure of shortest paths in graphs with edge weights that guarantee that the shortest path between any pair of vertices is unique. Motivated by the fact that a set of unique shortest paths is consistent in

\footnotetext{
* This research began at the Graph and Network Visualization Workshop 2019 (GNV'19) in Heiligkreuztal. S. C. is funded by the German Research Foundation DFG - Project-ID 50974019 - TRR 161 (B06). M. H. is supported by the Swiss National Science Foundation within the collaborative DACH project Arrangements and Drawings as SNSF Project 200021E-171681. S. K. is supported by NSF grants CCF-1740858, CCF-1712119, and DMS-1839274.
} 


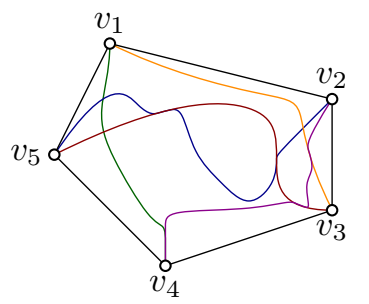

Fig. 1: A drawing of the geodetic graph $K_{5}$. It has a crossing formed by edges $v_{1} v_{3}$ and $v_{2} v_{5}$. In addition, edges $v_{1} v_{4}$ and $v_{2} v_{4}$ meet but do not cross since their meet includes vertex $v_{4}$. Finally, edges $v_{2} v_{5}$ and $v_{3} v_{5}$ meet twice violating the property of philogeodetic drawings.

the sense that no two such paths can "intersect, split apart, and then intersect again", he conjectured that if the shortest path between any pair of vertices in a graph is unique then the graph can be drawn so that any two shortest paths meet at most once. Formally, a meet of two Jordan curves $\gamma_{1}, \gamma_{2}:[0,1] \rightarrow \mathbb{R}^{2}$ is a pair of maximal intervals $I_{1}, I_{2} \subseteq[0,1]$ for which there is a bijection $\iota: I_{1} \rightarrow I_{2}$ so that $\gamma_{1}(x)=\gamma_{2}(\iota(x))$ for all $x \in I_{1}$. A crossing is a meet with $\left(I_{1} \cup I_{2}\right) \cap\{0,1\}=\emptyset$. Two curves meet $k$ times if they have $k$ pairwise distinct meets. For example, shortest paths in a simple polygon (geodesic paths) have the property that they meet at most once [6].

A drawing of a graph $G$ in $\mathbb{R}^{2}$ maps the vertices to pairwise distinct points and maps each edge to a Jordan arc between the two end-vertices that is disjoint from any other vertex. Drawings extend in a natural fashion to paths: Let $\varphi$ be a drawing of $G$, and let $P=v_{1}, \ldots, v_{n}$ be a path in $G$. Then let $\varphi(P)$ denote the Jordan arc that is obtained as the composition of the curves $\varphi\left(v_{1} v_{2}\right), \ldots, \varphi\left(v_{n-1} v_{n}\right)$. A drawing $\varphi$ of a graph $G$ is philogeodetic if for every pair $P_{1}, P_{2}$ of shortest paths in $G$ the curves $\varphi\left(P_{1}\right)$ and $\varphi\left(P_{2}\right)$ meet at most once.

An unweighted graph is geodetic if there is a unique shortest path between every pair of vertices. Trivial examples of geodetic graphs are trees and complete graphs. Observe that any two shortest paths in a geodetic graph are either disjoint or they intersect in a path. Thus, a planar drawing of a planar geodetic graph is philogeodetic. Also every straight-line drawing of a complete graph is philogeodetic. Refer to Fig. 1 for an illustration of a drawing of a complete graph that is not philogeodetic; this example also highlights some of the concepts discussed above. It is a natural question to ask whether every (geodetic) graph admits a philogeodetic drawing.

Results. We show that there exist geodetic graphs that require some pair of shortest paths to meet at least four times (Theorem 1). The idea is to start with a sufficiently large complete graph and subdivide every edge exactly twice. The Crossing Lemma [8] can be used to show that some pair of shortest paths must cross at least four times. By increasing the number of subdivisions per edge, we can reduce the density and obtain sparse counterexamples. The bound on the 
number of crossings is tight because any uniformly subdivided $K_{n}$ can be drawn so that every pair of shortest paths meets at most four times (Theorem 2).

On one hand, our construction yields counterexamples of diameter five. On the other hand, the unique graph of diameter one is the complete graph, which is geodetic and admits a philogeodetic drawing (e.g., any straight-line drawing since all unique shortest paths are single edges). Hence, it is natural to ask what is the largest $d$ so that every geodetic graph of diameter $d$ admits a philogeodetic drawing. We show that $d=1$ by exhibiting an infinite family of geodetic graphs of diameter two that do not admit philogeodetic drawings (Theorem 3). The construction is based on incidence graphs of finite affine planes. The proof also relies on the crossing lemma.

Geodetic graphs. Geodetic graphs were introduced by Ore who asked for a characterization as Problem 3 in Chapter 6 of his book "Theory of Graphs" [7, p. 104]. An asterisk flags this problem as a research question, which seems justified, as more than sixty years later a full characterization is still elusive.

Stemple and Watkins [14,15] and Plesník [10] resolved the planar case by showing that a connected planar graph is geodetic if and only if every block is (1) a single edge, (2) an odd cycle, or (3) stems from a $K_{4}$ by iteratively choosing a vertex $v$ of the $K_{4}$ and subdividing the edges incident to $v$ uniformly. Geodetic graphs of diameter two were fully characterized by Scapellato [12]. They include the Moore graphs [3] and graphs constructed from a generalization of affine planes. Further constructions for geodetic graphs were given by Plesník [10,11], Parthasarathy and Srinvasan [9], and Frasser and Vostrov [2].

Plesník [10] and Stemple [13] proved that a geodetic graph is homeomorphic to a complete graph if and only if it is obtained from a complete graph $K_{n}$ by iteratively choosing a vertex $v$ of the $K_{n}$ and subdividing the edges incident to $v$ uniformly. A graph is geodetic if it is obtained from any geodetic graph by uniformly subdividing each edge an even number of times $[9,11]$. However, the graph $G$ obtained by uniformly subdividing each edge of a complete graph $K_{n}$ an odd number of times is not geodetic: Let $u, v, w$ be three vertices of $K_{n}$ and let $x$ be the middle subdivision vertex of the edge $u v$. Then there are two shortest $x$-w-paths in $G$, one containing $v$ and one containing $u$.

\section{Subdivision of a Complete Graph}

The complete graph $K_{n}$ is geodetic and rather dense. However, all shortest paths are very short, as they comprise a single edge only. So despite the large number of edge crossings in any drawing, every pair of shortest paths meets at most once, as witnessed, for instance, by any straight-line drawing of $K_{n}$. In order to lengthen the shortest paths it is natural to consider subdivisions of $K_{n}$.

As a first attempt, one may want to "take out" some edge $u v$ by subdividing it many times. However, Stemple [13] has shown that in a geodetic graph every path where all internal vertices have degree two must be a shortest path. Thus, it is impossible to take out an edge using subdivisions. So we use a different approach instead, where all edges are subdivided uniformly. 
Theorem 1. There exists an infinite family of sparse geodetic graphs for which in any drawing in $\mathbb{R}^{2}$ some pair of shortest paths meets at least four times.

Proof. Take an even number $t$ and a complete graph $K_{s}$ for some $s \in \mathbb{N}$. Subdivide each edge $t$ times. The resulting graph $K(s, t)$ is geodetic. See Fig. 4 for a drawing of $K(8,2)$. Note that $K(s, t)$ has $n=s+t\left(\begin{array}{l}s \\ 2\end{array}\right)$ vertices and $m=(t+1)\left(\begin{array}{l}s \\ 2\end{array}\right)$ edges, with $m \in O(n)$, for $s$ fixed and $t$ sufficiently large. Consider a drawing $\Gamma$ of $K(s, t)$.

Let $B$ denote the set of $s$ branch vertices in $K(s, t)$, which correspond to the vertices of the original $K_{s}$. For two distinct vertices $u, v \in B$, let [uv] denote the shortest $u v$-path in $K(s, t)$, which corresponds to the subdivided edge $u v$ of the underlying $K_{s}$. As $t$ is even, the path [uv] consists of $t+1$ (an odd number of) edges. For every such path $[u v]$, with $u, v \in B$, we charge the crossings in $\Gamma$ along the $t+1$ edges of [uv] to one or both of $u$ and $v$ as detailed below; see Fig. 2 for illustration.

- Crossings along an edge that is closer to $u$ than to $v$ are charged to $u$;

- crossings along an edge that is closer to $v$ than to $u$ are charged to $v$; and

- crossings along the single central edge of $[u v]$ are charged to both $u$ and $v$.

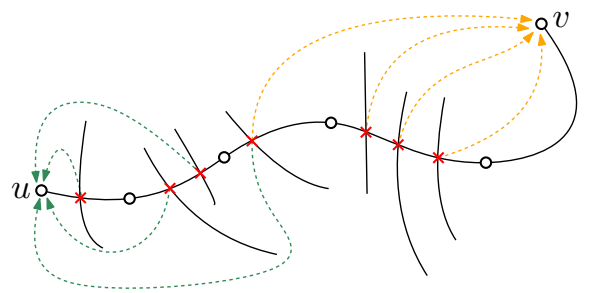

Fig. 2: Every crossing is charged to at least one endpoint of each of the two involved (independent) edges. Vertices are shown as white disks, crossings as red crosses, and charges by dotted arrows. The figure shows an edge $u v$ that is subdivided four times, splitting it into a path with five segments. A crossing along any such segment is assigned to the closest of $u$ or $v$. For the central segment, both $u$ and $v$ are at the same distance, and any crossing there is assigned to both $u$ and $v$.

Let $\Gamma_{s}$ be the drawing of $K_{s}$ induced by $\Gamma$ : every vertex of $K_{s}$ is placed at the position of the corresponding branch vertex of $K(s, t)$ in $\Gamma$ and every edge of $K_{s}$ is drawn as a Jordan arc along the corresponding path of $K(s, t)$ in $\Gamma$. Assuming $\left(\begin{array}{l}s \\ 2\end{array}\right) \geq 4 s$ (i.e., $s \geq 9$ ), by the Crossing Lemma [8], at least

$$
\frac{1}{64} \frac{\left(\begin{array}{l}
s \\
2
\end{array}\right)^{3}}{s^{2}}=\frac{1}{512} s(s-1)^{3} \geq c \cdot s^{4}
$$

pairs of independent edges cross in $\Gamma_{s}$, for some constant $c$. Every crossing in $\Gamma_{s}$ corresponds to a crossing in $\Gamma$ and is charged to at least two (and up to four) 
vertices of $B$. Thus, the overall charge is at least $2 c s^{4}$, and at least one vertex $u \in B$ gets at least the average charge of $2 c s^{3}$.

Each charge unit corresponds to a crossing of two independent edges in $\Gamma_{s}$, which is also charged to at least one other vertex of $B$. Hence, there is a vertex $v \neq u$ so that at least $2 c s^{2}$ crossings are charged to both $u$ and $v$. Note that there are only $s-1$ edges incident to each of $u$ and $v$, and the common edge $u v$ is not involved in any of the charged crossings (as adjacent rather than independent edge). Let $E_{x}$, for $x \in B$, denote the set of edges of $K_{s}$ that are incident to $x$.

We claim that there are two pairs of mutually crossing edges incident to $u$ and $v$, respectively; that is, there are sets $C_{u} \subset E_{u} \backslash\{u v\}$ and $C_{v} \subset E_{v} \backslash\{u v\}$ with $\left|C_{u}\right|=\left|C_{v}\right|=2$ so that $e_{1}$ crosses $e_{2}$, for all $e_{1} \in C_{u}$ and $e_{2} \in C_{v}$.

Before proving this claim, we argue that establishing it completes the proof of the theorem. By our charging scheme, every crossing $e_{1} \cap e_{2}$ happens at an edge of the path $\left[e_{1}\right]$ in $\Gamma$ that is at least as close to $u$ as to the other endpoint of $e_{1}$. Denote the three vertices that span the edges of $C_{u}$ by $u, x, y$. Consider the two subdivision vertices $x^{\prime}$ along $[u x]$ and $y^{\prime}$ along $[u y]$ that form the endpoint of the middle edge closer to $x$ and $y$, respectively, than to $u$; see Fig. 3 for illustration.

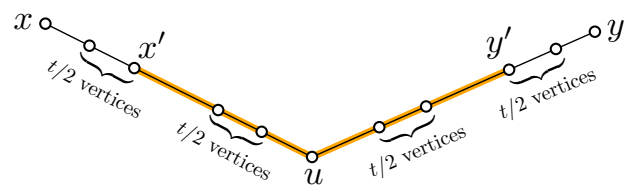

Fig. 3: Two adjacent edges $u x$ and $u y$, both subdivided $t$ times, and the shortest path between the "far" endpoints $x^{\prime}$ and $y^{\prime}$ of the central segments of $[u x]$ and [uy].

The triangle $u x y$ in $K_{s}$ corresponds to an odd cycle of length $3(t+1)$ in $K(s, t)$. So the shortest path between $x^{\prime}$ and $y^{\prime}$ in $K(s, t)$ has length $2(1+t / 2)=$ $t+2$ and passes through $u$, whereas the path from $x^{\prime}$ via $x$ and $y$ to $y^{\prime}$ has length $3(t+1)-(t+2)=2 t+1$, which is strictly larger than $t+2$ for $t \geq 2$. It follows that the shortest path between $x^{\prime}$ and $y^{\prime}$ in $K(s, t)$ is crossed by both edges in $C_{v}$. A symmetric argument yields two subdivision vertices $a^{\prime}$ and $b^{\prime}$ along the two edges in $C_{v}$ so that the shortest $a^{\prime} b^{\prime}$-path in $K(s, t)$ is crossed by both edges in $C_{u}$. By definition of our charging scheme (that charges only "nearby" crossings to a vertex), the shortest paths $x^{\prime} y^{\prime}$ and $a^{\prime} b^{\prime}$ in $K(s, t)$ have at least four crossings.

It remains to prove the claim. To this end, consider the bipartite graph $X$ on the vertex set $E_{u} \cup E_{v}$ where two vertices are connected if the corresponding edges are independent and cross in $\Gamma_{s}$. Observe that two sets $C_{u}$ and $C_{v}$ of mutually crossing pairs of edges (as in the claim) correspond to a 4-cycle $C_{4}$ in $X$. So suppose for the sake of a contradiction that $X$ does not contain $C_{4}$ as a subgraph. Then by the Kövári-Sós-Turán Theorem [5] the graph $X$ has $O\left(s^{3 / 2}\right)$ edges. But we already know that $X$ has at least $2 c s^{2}=\Omega\left(s^{2}\right)$ edges, which yields a contradiction. Hence, $X$ is not $C_{4}$-free and the claim holds. 
The bound on the number of crossings in Theorem 1 is tight.

Theorem 2. A graph obtained from a complete graph by subdividing the edges uniformly an even number of times can be drawn so that every pair of shortest paths crosses at most four times.

Proof (Sketch). Place the vertices in convex position. Draw the subdivided edges along straight-line segments. For each edge, put half of the subdivision vertices very close to one endpoint and the other half very close to the other endpoint (Fig. 4). As a result, all crossings fall into the central segment of the path.

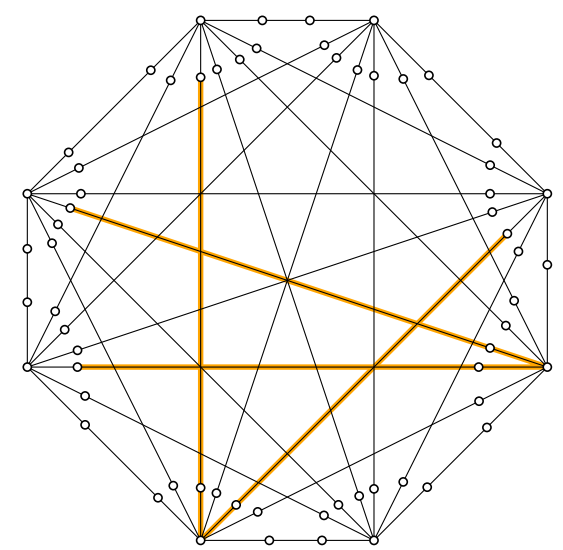

Fig. 4: A drawing of $K(8,2)$, the complete graph $K_{8}$ where every edge is subdivided twice, so that every pair of shortest paths meets at most four times. Two shortest paths that meet four times are shown bold and orange.

\section{Graphs of Diameter Two}

In this section we give examples of geodetic graphs of diameter two that cannot be drawn in the plane such that any two shortest paths meet at most once.

An affine plane of order $k \geq 2$ consists of a set of lines and a set of points with a containment relationship such that (i) each line contains $k$ points, (ii) for any two points there is a unique line containing both, (iii) there are three points that are not contained in the same line, and (iv) for any line $\ell$ and any point $p$ not on $\ell$ there is a line $\ell^{\prime}$ that contains $p$, but no point from $\ell$. Two lines that do not contain a common point are parallel. Observe that each point is contained in $k+1$ lines. Moreover, there are $k^{2}$ points and $k+1$ classes of parallel lines each containing $k$ lines. The 2-dimensional vector space $\mathbb{F}^{2}$ over a finite field $\mathbb{F}$ of order $k$ with the lines $\{(x, m x+b) ; x \in \mathbb{F}\}, m, b \in \mathbb{F}$ and $\left\{\left(x_{0}, y\right) ; y \in \mathbb{F}\right\}$, 
$x_{0} \in \mathbb{F}$ is a finite affine plane of order $k$. Thus, there exists a finite affine plane of order $k$ for any $k$ that is a prime power (see, e.g., [4]).

Scapellato [12] showed how to construct geodetic graphs of diameter two as follows: Take a finite affine plane of order $k$. Let $L$ be the set of lines and let $P$ be the set of points of the affine plane. Consider now the graph $G_{k}$ with vertex set $L \cup P$ and the following two types of edges: There is an edge between two lines if and only if they are parallel. There is an edge between a point and a line if and only if the point lies on the line; see Fig. 5. There are no edges between points. It is easy to check that $G_{k}$ is a geodetic graph of diameter two.

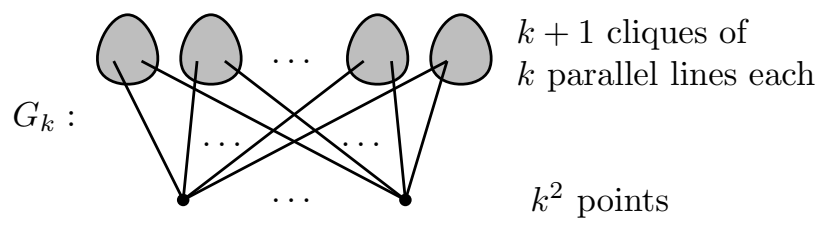

Fig. 5: Structure of the graph $G_{k}$.

Theorem 3. There are geodetic graphs of diameter two that cannot be drawn in the plane such that any two shortest paths meet at most once.

Proof. Let $k \geq 129$ be such that there exists an affine plane of order $k$ (e.g., the prime $k=131$ ). Assume there was a drawing of $G_{k}$ in which any two shortest paths meet at most once. Let $G$ be the bipartite subgraph of $G_{k}$ without edges between lines. Observe that any path of length two in $G$ is a shortest path in $G_{k}$. As $G$ has $n=2 k^{2}+k$ vertices and $m=k^{2}(k+1)>k n / 2$ edges, we have $m>4 n$, for $k \geq 8$. Therefore, by the Crossing Lemma [8, Remark 2 on p. 238] there are at least $m^{3} / 64 n^{2}>k^{3} n / 512$ crossings between independent edges in $G$.

Hence, there is a vertex $v$ such that the edges incident to $v$ are crossed more than $k^{3} / 128$ times by edges not incident to $v$. By assumption, (a) any two edges meet at most once, (b) any edge meets any pair of adjacent edges at most once, and (c) any pair of adjacent edges meets any pair of adjacent edges at most once. Thus, the crossings with the edges incident to $v$ stem from a matching. It follows that there are at most $(n-1) / 2=\left(2 k^{2}+k-1\right) / 2$ such crossings. However, $\left(2 k^{2}+k-1\right) / 2<k^{3} / 128$, for $k \geq 129$.

\section{Open Problems}

We conclude with two open problems: (1) Are there diameter-2 geodetic graphs with edge density $1+\varepsilon$ that do not admit a philogeodetic drawing? (2) What is the complexity of deciding if a geodetic graph admits a philogeodetic drawing? 


\section{References}

1. Bodwin, G.: On the structure of unique shortest paths in graphs. In: Proceedings of the Thirtieth Annual ACM-SIAM Symposium on Discrete Algorithms (SODA'19). pp. 2071-2089. SIAM (2019), https://doi.org/10.1137/1.9781611975482.125

2. Frasser, C.E., Vostrov, G.N.: Geodetic graphs homeomorphic to a given geodetic graph. CoRR abs/1611.01873 (2016), http://arxiv.org/abs/1611.01873

3. Hoffman, A.J., Singleton, R.R.: Moore graphs with diameter 2 and 3. IBM Journal of Research and Development 5(4), 497-504 (1960), doi:10.1147/rd.45.0497

4. Hughes, D.R., Piper, F.C.: Projective Planes, Graduate Texts in Mathematics, vol. 6. Springer (1973)

5. Kővári, T., Sós, V.T., Turán, P.: On a problem of K. Zarankiewicz. Colloq. Math. 3, 50-57 (1954), https://doi.org/10.4064/cm-3-1-50-57

6. Lee, D.T., Preparata, F.P.: Euclidean shortest paths in the presence of rectilinear barriers. Networks 14, 393-410 (1984), https://doi.org/10.1002/net. 3230140304

7. Ore, Ø.: Theory of Graphs, Colloquium Publications, vol. 38. American Mathematical Society, Providence, RI (1962), https://dx.doi.org/10.1090/coll/038

8. Pach, J., Tóth, G.: Which crossing number is it anyway? J. Combin. Theory Ser. B 80(2), 225-246 (2000), https://doi.org/10.1006/jctb.2000.1978

9. Parthasarathy, K.R., Srinivasan, N.: Some general constructions of geodetic blocks. J. Combin. Theory Ser. B 33(2), 121-136 (1982), https://doi.org/10.1016/ 0095-8956 (82) 90063-6

10. Plesník, J.: Two constructions of geodetic graphs. Mathematica Slovaca 27(1), 65-71 (1977), https://dml.cz/handle/10338.dmlcz/136134

11. Plesník, J.: A construction of geodetic graphs based on pulling subgraphs homeomorphic to complete graphs. J. Combin. Theory Ser. B 36(3), 284-297 (1984), https ://doi .org/10.1016/0095-8956(84)90034-0

12. Scapellato, R.: Geodetic graphs of diameter two and some related structures. J. Combin. Theory Ser. B 41(2), 218-229 (1986), https://doi.org/10.1016/ 0095-8956 (86) 90045-6

13. Stemple, J.G.: Geodetic graphs homeomorphic to a complete graph. Annals of the New York Academy of Science 319(1), 512-517 (1979), https://doi .org/10. 1111/j.1749-6632.1979.tb32829.x

14. Stemple, J.G., Watkins, M.E.: On planar geodetic graphs. J. Combin. Theory Ser. B 4(2), 101-117 (1968), https://doi.org/10.1016/S0021-9800(68)80035-3

15. Watkins, M.E.: A characterization of planar geodetic graphs. J. Combin. Theory 2(1), 102-103 (1967), https://doi.org/10.1016/S0021-9800 (67)80118-2 


\section{A Proof of Theorem 2}

Proof. Draw the graph as described on Page 6 and as illustrated in Fig. 4 for $K(8,2)$. There are two different types of vertices, and six different types of shortest paths. Let $B$ denote the set of branch vertices, and let $S$ denote the set of subdivision vertices. Note that for every edge $u v$ of $K_{n}$, only the central segment of the subdivided path $[u v]$ may have crossings in the drawing. We claim that every shortest path in the graph contains at most two central segments in the drawing, from which the theorem follows immediately. Consider a pair $u, v$ of vertices.

Case 1: $\{u, v\} \cap B \neq \emptyset$. Suppose without loss of generality that $u \in B$. If $v \in B$ or $v \in S$ subdivides an edge incident to $u$, then the shortest $u v$-path contains at most one central segment. Otherwise, $v \in S$ subdivides an edge $x y$ disjoint from $u$. One of $x$ or $y$, without loss of generality $x$ is closer to $v$. Then the shortest $u v$-path is $[v x][x u]$, which contains exactly one central segment, $[x u]$.

Case 2: $u, v \in S$. If $u$ and $v$ subdivide the same edge, then the shortest $u v$ path contains at most one central segment. If $u$ and $v$ subdivide distinct adjacent segments, $x y$ and $x z$, then the shortest $u v$-path is either $[u x][x v]$, which contains at most two central segments. Or the sum of the length of $[u y]$ and $[z v]$ is at most half of the number of subdivision vertices per edge and the shortest $u v$-path is $[u y][y z][z v]$, which then contains at most one central segment. Otherwise, $u$ and $v$ subdivide disjoint segments, $x y$ and $w z$, where without loss of generality $x$ is closer to $u$ than $y$ and $w$ is closer to $v$ than $z$. Then the shortest $u v$-path is $[u x][x w][w v]$, which contains exactly one central segment, $[x w]$.

\section{B Proof that $G_{k}$ (as Defined in Section 3) is Geodetic}

The following statement follows from Scapellato's classification [12]. As we need much less than this classification in its full generality, we provide an easy proof of what we use, for the sake of self-containment.

Lemma 1. $G_{k}$ is a geodetic graph of diameter two.

Proof. Two lines have distance one if they are parallel. Otherwise they share exactly one vertex and, hence, are connected by exactly one path of length two. For any two points there is exactly one line that contains both. Given a line $\ell$ and a point $p$ then either $p$ lies on $\ell$ and, thus, $p$ and $\ell$ have distance one. Or there is exactly one line $\ell^{\prime}$ containing $p$ that is parallel to $\ell$ and, thus, there is exactly one path of length two between $\ell$ and $p$. 